\title{
Neutropenic sepsis: a potentially life-threatening complication of chemotherapy
}

\author{
Authors: Amy Ford ${ }^{A}$ and Ernie Marshall ${ }^{B}$
}

\section{Background}

Neutropenic sepsis (NS) is defined by the National Institute for Health and Care Excellence (NICE) as a temperature of greater than $38^{\circ} \mathrm{C}$, with a neutrophil count of less than $0.5 \times 10^{9} / 1$ in a patient undergoing systemic anticancer therapy (SACT). ${ }^{1}$ Nevertheless, there is considerable variation in symptom presentation and clinicians should suspect NS in all patients undergoing chemotherapy who become unwell, whether they have a fever or not. NS most commonly follows the delivery of cytotoxic chemotherapy with a typical neutrophil nadir occurring at days 10-14. Newer, biological systemic anti-cancer treatments and radiotherapy have a much lower propensity to cause neutropenia. Therapy for haematological malignancies may result in a deeper nadir and greater duration of neutropenia and hence a relatively high rate of febrile neutropenia.

NS continues to be a significant cause of cancer-treatmentrelated mortality; the number of attributable deaths has doubled over the past decade, even after adjusting for the increasing number of cancers diagnosed during this period. ${ }^{1}$ The majority of NS deaths are in the elderly (aged 65-79 years) and are in part related to the increasing use of chemotherapy, greater dose intensity and greater comorbidities. Importantly, the rising mortality rate may also reflect an increasing element of devolved and dislocated care associated with current service design, as highlighted by the National Clinical Enquiry into Patient Deaths (NCEPOD). The report evaluated deaths within 30 days of receiving SACT and identified evidence of dislocation of care, delayed treatment and lack of patient engagement as contributory factors. ${ }^{2}$ Patients need to be aware of the signs, symptoms and risks of NS and the need to seek medical advice as soon as possible. The National Clinical Enquiry has resulted in the evolution of acute oncology services nationally and the development of a NICE clinical guideline for the prevention and management of NS. ${ }^{1}$

Sepsis during periods of neutropenia is typically associated with bacteraemia in the absence of localising signs. Blood cultures are important in guiding subsequent therapy but

Authors: Aspecialist registrar in medical oncology, Clatterbridge Cancer Centre, Bebington, UK; ${ }^{\mathrm{B} M a c m i l l a n}$ consultant in medical oncology, Clatterbridge Cancer Centre, Bebington, UK they are often negative. The pattern of causative organisms in febrile neutropenia has evolved over recent decades. Several decades ago, NS was largely caused by Gramnegative pathogens. In the 1980s, however, indwelling plastic catheters, which promote the colonisation of Grampositive skin flora and their entry into the bloodstream, were introduced. As a consequence, drug-resistant Gram-positive organisms, such as methicillin-resistant Staphylococcus aureus (MRSA) and vancomycin-resistant Enterococcus (VRE), have become increasingly prevalent. ${ }^{3}$ Gram-negative organisms, in particular Klebsiella species and E coli strains, are also implicated in febrile neutropenia, and antibiotic resistance resulting from extended spectrum $\beta$-lactamase (ESBL) production is increasing in these organisms. ${ }^{3}$

\section{Prevention}

The predictable consequences of cytotoxic chemotherapy suggest that there is a role for preventative action, including both patient education and therapeutics. Primary antibiotic prophylaxis, using fluoroquinolones or cotrimoxazole (delivered before an episode of neutropenic sepsis), reduces the incidence of NS and short-term mortality. ${ }^{4}$ Nevertheless, this approach needs to be balanced against the potential risks of increasing antibiotic resistance and the adverse effects of antibiotic use. NICE guidelines recommend the use of prophylactic quinolones for the predicted duration of neutropenia only for patients undergoing stem cell transplants, or for those being treated for acute leukaemias or for solid tumours where significant neutropenia (neutrophil count less than $\left.0.5 \times 10^{9} / 1\right)$ is anticipated. ${ }^{1}$

The administration of leucocyte growth factors (granulocyte and granulocyte-macrophage colony stimulating factor [G-CSF]) reduces the severity and duration of neutropenia, the rate of NS and possibly the hospital length of stay. Despite this, there is no convincing evidence that prophylaxis with G-CSF reduces short-term mortality. ${ }^{5}$ The efficacy of G-CSF may vary according to the type of cancer therapy (treatments for leukaemia, lymphomas or solid tumours, or stem cell transplants) and must be weighed against the side effects of its use, which include bone pain, headache and nausea. NICE guidelines advocate against the routine use of G-CSF, unless it is an integral part of a specific chemotherapy regime. International guidelines recommend the use of G-CSF in selected patients who have a risk of NS exceeding $20 \% \cdot{ }^{6,7}$ 


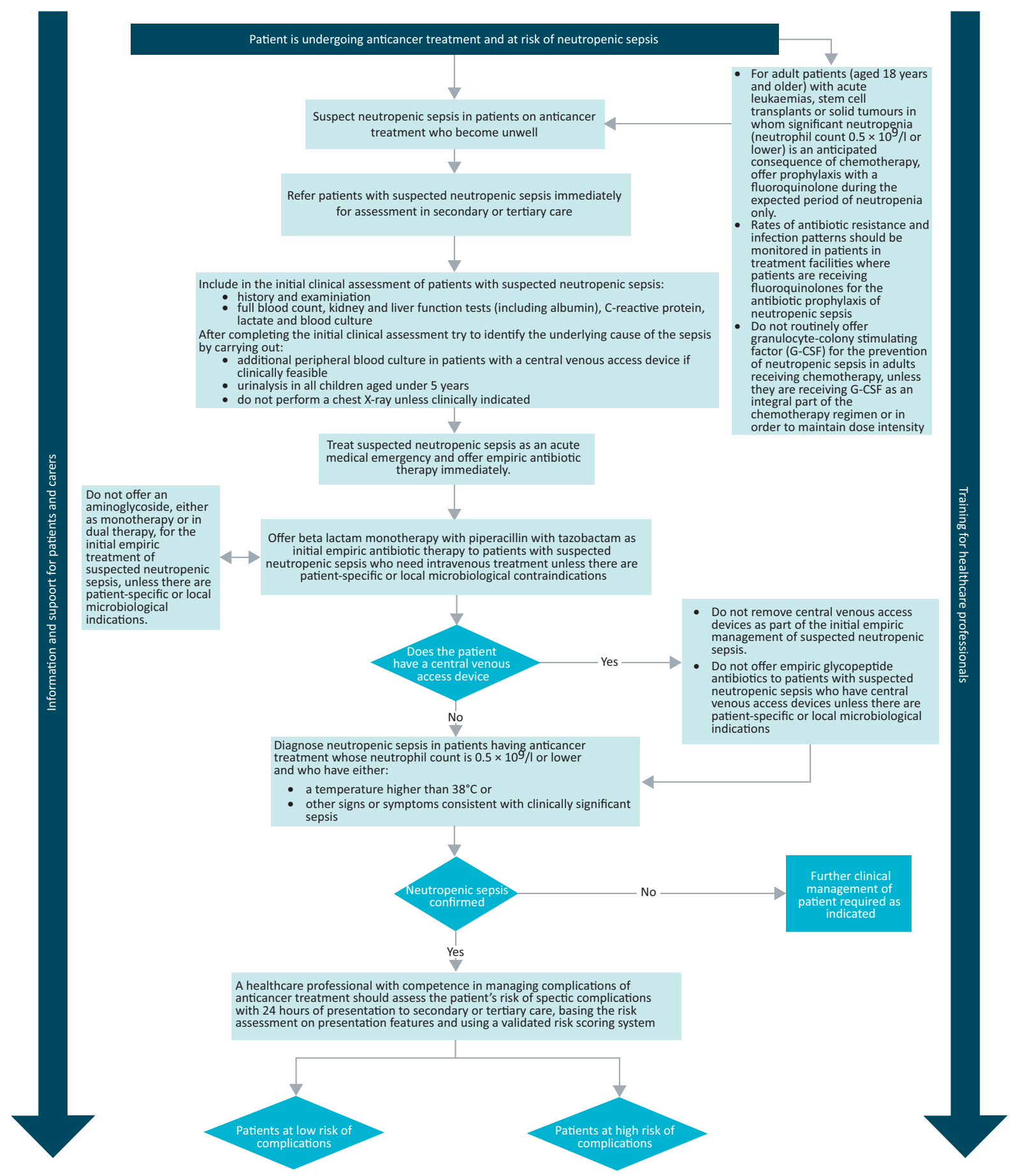

Fig 1. Management algorithm from the National Institute of Health and Care Excellence (NICE) guidelines. ${ }^{1}$ Reproduced with permission. The full version is available at: www.nice.org.uk/guidance/cg151

\section{Evaluation of neutropenic sepsis}

Patients who present with a fever shortly after anti-cancer treatment should be promptly assessed by professionals who are familiar with NS and who have rapid access to broad-spectrum antibiotics. Emergency oncological assessment should follow the principles outlined in the NICE guidelines ${ }^{1}$ (Fig 1) and in the Acute Care

Toolkit series $7 .{ }^{8}$ Initial assessment, by way of a thorough history and examination, should be supplemented by a clear understanding of the patient's cancer journey with particular reference to the details and dates of their SACT. All patients undergoing chemotherapy should have access to a chemotherapy alert card or patient-held record that outlines immediate treatment plans and key contact details for oncology triage. Investigations should include full blood count $(\mathrm{FBC})$, renal function, liver function, C-reactive protein, 




Fig 2. Risk-stratified care.

lactate and blood cultures. Where a central venous catheter is in use, peripheral blood cultures should also be obtained. Urinalysis, chest X-ray, stool, sputum and cerebrospinal fluid culture are rarely helpful and should only be undertaken when clinically indicated. The differential diagnoses to be considered include malignancyrelated fever, pulmonary embolism and chemotherapy-induced fever (most commonly seen with bleomycin).

Because of the potential risks of missing the diagnosis of NS, any patient who has undergone chemotherapy within 6 weeks of presentation should be considered as septic until proven otherwise. Not uncommonly, NS may present with ill-defined symptoms such as confusion and gastrointestinal upset in the absence of fever. All hospitals with an accident and emergency department should ensure that links are established with local acute oncology services to facilitate the development of a management pathway for NS, which should incorporate immediate administration of broad spectrum antibiotics and early review by a member of the oncology team. ${ }^{9}$

\section{Risk stratification}

Although NS should be considered a medical emergency, only a minority of patients will develop life-threatening infections or will suffer other serious complications. Consequently, patients with NS are increasingly stratified into those at high- and low-risk of septic complications, the latter having the potential for treatment with oral antibiotics and early discharge (Fig 2). Stratification is based on presenting signs and symptoms, the nature of the underlying malignancy and existing comorbidities, and should be undertaken using a validated risk-scoring tool, such as the Multinational Association for Supportive Care in Cancer (MASCC) risk index (Table 1). ${ }^{10,11}$ The burden of illness (the first characteristic used to calculate the risk index) represents a measure of how unwell the patient is at presentation, but lacks objective definition. Clinical experience is needed to inform this judgement and it is recommended that risk stratification be undertaken by a healthcare professional with experience in managing the complications of anti-cancer treatment. ${ }^{1}$

\section{Table 1. MASCC Index.}

\section{Characteristic}

Burden of illness:

Either, no or mild symptoms*

or moderate symptoms*

No hypotension

5

No chronic obstructive pulmonary disease

Solid tumour/lymphoma or no previous fungal infection

No dehydration

Outpatient status at onset of fever

Aged $<60$ years theoretical score is therefore 26 . A threshold $\geq 21$ points defines 'low risk'. MASCC $=$ Multinational Association for Supportive Care in Cancer. 


\section{Key points}

Neutropenic sepsis is a significant cause of cancer-related mortality, requiring prompt diagnosis and treatment with empirical antibiotic treatment

Any patient receiving systemic anti-cancer therapy should be evaluated and treated as septic in origin until proven otherwise

Risk-stratification tools such as the MASCC index are central to avoiding the overtreatment of low-risk patients and the early and appropriate treatment of high-risk patients

Patients need to be aware of the signs and symptoms of neutropenic sepsis, and of the importance of having access to a thermometer at home

Acute oncology teams have an important role in optimising the management of neutropenic sepsis outside of specialist oncology centres

KEYWORDS: Antibiotics, chemotherapy, neutropenic sepsis, MASCC Index, risk stratification

\section{Management of neutropenic sepsis}

In hospitals where professionals are familiar with risk stratification, low-risk patients should be considered for treatment with oral antibiotics followed by early discharge, taking into account their social circumstances. The minimum safe period of observation prior to discharge has yet to be determined, but most studies to date have observed patients for at least 24 hours before discharge. ${ }^{3}$ Any early discharge strategy should be linked with robust follow up and continuous access to expert cancer triage. Intravenous antibiotics are warranted where specific expertise is lacking or if coexisting complications of chemotherapy, such as vomiting or severe mucositis, prevent the administration of oral medication. High-risk patients should receive empirical intravenous antibiotics without delay on the basis of the initial clinical concern and not blood-count results. The National Cancer Peer Review Programme recommends a national target time of 1 hour from the point at which a likely diagnosis of NS is made (on the basis of clinical assessment rather than laboratory results) to administration of antibiotic therapy. ${ }^{12}$

\section{First-line antibiotics}

When choosing empirical antibiotics, the epidemiological spectrum of bloodstream isolates and regional patterns of antibiotic resistance should be considered. Treatment should follow local guidelines. In the absence of patient-specific or local microbiological contraindications, NICE guidance recommends $\beta$-lactam monotherapy using piperacillin with tazobactam as initial empirical treatment. It advises against the use of aminoglycosides in this context, as there is no evidence that combined therapy reduces mortality. Monotherapy is also associated with fewer adverse effects, such as nephrotoxicity, and avoids the need to monitor aminoglycocide levels. ${ }^{1}$

\section{Ongoing care}

Following initial diagnosis and management by acute care services, ongoing patient care should be coordinated by physicians who have expertise in the management of cancer-related sepsis and supported by acute oncology teams. Intravenous antibiotics may be replaced by oral antibiotics after 48 hours if the risk of developing septic complications is reassessed as low. ${ }^{1}$ In the setting of solid tumours, antibiotics should be discontinued in patients whose NS has responded to treatment, as evidenced by lysis of fever and subjective and objective improvement, irrespective of neutrophil count. ${ }^{1}$ Where an organism has been isolated, treatment should be continued for a minimum of 5 days. If there is no strong clinical suspicion of central-line infection, there is no need for removal in the initial phase of management, but this should be reviewed if there is no resolution of fever or if there is evidence of post-flushing fever. ${ }^{1}$

Persistent fever, in the absence of clinical deterioration or new focal signs, is not an indication for switching antibiotic therapy unless guided by culture results. ${ }^{1,3}$ In the absence of a source of bacterial infection, patients with a persistent fever after 4-7 days, who are expected to be neutropenic for longer than 7 days, should be considered for empirical antifungal therapy and investigation for invasive fungal infections. ${ }^{3}$ Choice of empirical antifungal agent, if indicated, will depend on whether or not the patient has already received prophylactic antifungal treatment. ${ }^{3}$

Despite the advent of novel anticancer therapies, NS will continue to represent a common oncological emergency presentation to acute medical services. Reduction in mortality will require greater vigilance and awareness of sepsis in cancer patients, combined with early access to broad-spectrum antibiotics and effective coordination of care in the emergency setting.

\section{References}

1 National Institute for Health and Care Excellence. Neutropenic sepsis: prevention and management of neutropenic sepsis in cancer patients. CG151. London: NICE, 2012. www.nice.org.uk/guidance/cg151/ [Accessed 5 August 2014].

2 National Confidential Enquiry into Patient Outcome and Death, 2008. For better, for worse? A review of the care of patients who died within 30 days of receiving systemic anti-cancer therapy. London: NCEPOD, 2008. www.ncepod.org.uk/2008report3/Downloads/ SACT_report.pdf [Accessed 5 August 2014].

3 Freifeld AG, Bow EJ, Sepkowitz KA et al. Clinical practice guideline for the use of antimicrobial agents in neutropenic patients with cancer: 2010 update by the Infectious Diseases Society of America. Clin Infect Dis 2011;52:e56-e93.

4 Gafter-Gvili A, Fraser A, Paul M et al. Antibiotic prophylaxis for bacterial infections in afebrile neutropenic patients following chemotherapy. Cochrane Database Syst Rev 2012;1:CD004386.

5 Cooper K, Madan J, Whyte S et al. Granulocyte colony-stimulating factors for febrile neutropenia prophylaxis following chemotherapy: systematic review and meta-analysis. BMC Cancer 2011;11:404.

6 Aapro MS, Bohlius J, Cameron DA et al. 2010 update of EORTC guidelines for the use of granulocyte-colony stimulating factor to reduce the incidence of chemotherapy-induced febrile neutropenia in adult patients with lymphoproliferative disorders and solid tumours. Eur J Cancer 2011;47:8-32. 
7 Crawford J, Armitage J, Balducci L et al. Myeloid growth factors. J Natl Compr Canc Netw 2013;11:1266-90.

8 Royal College of Physicians. Acute care toolkit 7. Acute oncology on the acute medical unit. London: RCP, 2013. www.rcplondon.ac.uk/sites/ default/files/acute_care_toolkit_7.pdf [Accessed 5 August 2014].

9 NHS National Cancer Action Team. National Cancer Peer Review Programme. Manual for cancer services: acute oncology - including metatastic spinal cord compression measures (Version 1.0). London: National Cancer Action Team, 2011. www.gov.uk/government/ uploads/system/uploads/attachment_data/file/216121/dh_125889.pdf [Accessed 5 August 2014].

10 Klastersky J, Paesmans M, Edward EB et al. The Multinational Association for Supportive Care in Cancer risk index: a multinational scoring system for identifying low risk febrile neutropenic cancer patients. J Clin Oncol 2000;18:3038-51.
11 Innes H, Lim S, Hall A et al. Management of febrile neutropenia in solid tumours and lymphomas using the Multinational Association for Supportive Care in Cancer (MASCC) risk index: feasibility and safety in routine clinical practice. Support Care Cancer 2008;16:485-91.

12 National Chemotherapy Advisory Group. Chemotherapy services in England: ensuring quality and safety. London: National Chemotherapy Advisory Group, 2009. http://webarchive.nationalarchives.gov.uk/ 20130107105354/http://www.dh.gov.uk/prod_consum_dh/groups/ dh_digitalassets/documents/digitalasset/dh_104501.pdf [Accessed 5 August 2014].

Address for correspondence: Dr E Marshall,

Clatterbridge Cancer Centre, Clatterbridge Road, Bebington, Wirral CH63 4JY.

Email: emarshall@nhs.net

\title{
Metastatic spinal cord compression: a rare but important complication of cancer
}

\author{
Author: Peter Robson ${ }^{A}$
}

\section{Background}

Metastatic spinal cord compression (MSCC) is a well-recognised complication of cancer and usually presents as an oncological emergency. Metastases to the spine occur in 3-5\% of all patients who have cancer and are more common in patients with breast, prostate and lung cancer, in whom the incidence is $19 \% .^{1}$ The true incidence of MSCC is unknown but estimates are around $15 \%$ of patients with advanced cancer. ${ }^{2}$

MSCC is usually caused by the collapse or compression of a vertebral body that contains metastatic disease, but can also rarely be caused by direct tumour extension into the vertebral column. Compression of the cord initially causes oedema, venous congestion and demyelination, which are reversible. Prolonged compression leads to vascular injury, cord necrosis and permanent damage. Patients who have no neurological function for more than 48 hours are unlikely to improve. Their condition should be discussed with their primary tumour site clinician or oncologist before considering magnetic resonance imaging (MRI) or transfer. ${ }^{3}$

Author: ${ }^{A}$ consultant clinical oncologist, The Clatterbridge Cancer Centre NHS Foundation Trust, Bebington, UK

\section{Clinical symptoms and signs}

Back pain occurs in $95 \%$ of patients who present with MSCC. It is frequently the first symptom and often present for a prolonged period. The pain may present as spinal pain or radicular pain, and studies show that it is often of high intensity $(8 / 10)$. The next most common symptom of MSCC is limb weakness with many patients unable to walk unaided at presentation. ${ }^{3}$ Many patients who are at the point of developing MSCC are very unwell, have significant comorbidities or are of very poor performance status. Consideration should be made as to whether the patient would be fit enough for transfer and treatment before arranging an MRI. For a patient who is too frail or unfit for treatment, local palliative care is a more appropriate outcome; advice can be sought from the local acute oncology team or visiting oncologist.

Red flags for MSCC are: ${ }^{1}$

$>$ limb weakness

$>$ difficulty walking

$>$ sensory loss

$>$ bladder or bowel dysfunction

$>$ neurological signs

$>$ thoracic or cervical pain

$>$ pain that is increased by straining

$>$ nocturnal spinal pain. 